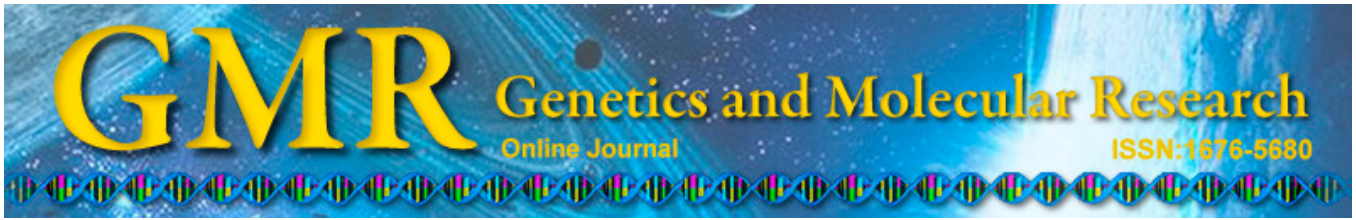

\title{
Potential of berberine to enhance antimicrobial activity of commonly used antibiotics for dairy cow mastitis caused by multiple drug-resistant Staphylococcus epidermidis infection
}

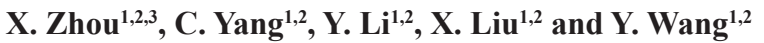 \\ ${ }^{1}$ Key Laboratory of Ministry of Education for Conservation and Utilization of \\ Special Biological Resources in the Western China, Yinchuan, China \\ ${ }^{2}$ College of Life Science, Ningxia University, Yinchuan, Ningxia, China \\ ${ }^{3}$ Environment and Biotechnology Center, Faculty of Life and Social Sciences, \\ Swinburne University of Technology, Victoria, Australia \\ Corresponding author: Y. Wang \\ E-mail: wyj@nxu.edu.cn
}

Genet. Mol. Res. 14 (3): 9683-9692 (2015)

Received December 12, 2014

Accepted May 26, 2015

Published August 19, 2015

DOI http://dx.doi.org/10.4238/2015.August.19.1

\begin{abstract}
Berberine is a plant alkaloid with antimicrobial activity against a variety of microorganisms. In this study, the antimicrobial properties of berberine against multi-drug resistant field isolates of Staphylococcus epidermidis were investigated using berberine alone or in combination with a commonly used antibiotics in veterinary clinics, including penicillin, lincomycin, and amoxicillin. The results indicated that the minimum inhibitory concentrations of berberine, penicillin, lincomycin, and amoxicillin against field $S$. epidermidis isolates were $2-512,0.8-213,0.4-1024$, and $0.4-256 \mu \mathrm{g} / \mathrm{mL}$, respectively. Furthermore, the synergistic effects of antimicrobial activity against these multi-drug resistant isolates were observed when the berberine was combined with penicillin, lincomycin, or amoxicillin; no antagonistic effect of the combination was detected in any of the clinical isolates.
\end{abstract}


These observations were further confirmed using a time-killing assay, in which a combination of 2 agents yielded a greater than 2.03-2.44 $\log _{10}$ decrease in colony-forming unit/mL compared with each agent alone. These findings suggest that berberine is a promising compound for preventing and treating multi-drug resistant S. epidermidis infected mastitis in dairy cows either alone or in combination with other commonly used antibiotics, such as penicillin, lincomycin, and amoxicillin.

Key words: Antibiotics; Berberine; Dairy cow mastitis; Staphylococcus epidermidis

\section{INTRODUCTION}

Staphylococcus epidermidis is often considered to be a non-pathogenic bacterium, but it is a leading cause of hospital-acquired infections and is the most common pathogen associated with infections of surgical implants and other prosthetic devices in the hospital because of its adhesion properties and ability to form a biofilm on biomaterial surfaces (Gomes et al., 2012; Mekni et al., 2012). In veterinary practice, it is also one of the most prevalent species of coagulase-negative staphylococci (CNS) isolated from mastitis in dairy cows (Koop et al., 2012; Piessens et al., 2012). CNS is a group of the most frequently isolated microorganisms in cows and heifers in herds and the milk of goats and cows and is considered to be an emerging pathogen of bovine mastitis (Pyörälä and Taponen, 2009; FernándezRufete et al., 2012).

In animal clinical practice, variability in antimicrobial susceptibility to CNS, along with an increasing number of methicillin-resistant strains, has been observed, making it difficult to select an effective antibiotic for preventing and treating mastitis. Thus, individual susceptibility must be assessed to identify the optimal antibiotic treatment for mastitis (Zadoks et al., 2011). Previous studies using intramammary injection or systemic administration of penicillin, pirlimycin, or florfenicol have demonstrated a herd-level association between antimicrobial resistance and the use of antibiotics for $S$. aureus under field conditions (Saini et al., 2012). Recently, molecular epidemiological studies on mastitis pathogens and methicillin-resistant $S$. aureus (MRSA) in bovines and humans has revealed that the pathogens may not only share virulence factors, but also have distinct virulence factors that appear to be important in host adaptation. The exchange of genes encoding these virulence factors between strains may have a great impact on public health (Holmes and Zadoks, 2011; Zadoks et al., 2011). With the emergence of antibiotic-resistant bacterial isolates found in the mastitis of dairy livestock (Nickerson, 2009), as well as the increasing evidence of the S. epidermidis-related mastitis pathogen (Oliveira et al., 2006), identifying or developing a novel agent or regimen for preventing or treating of mastitis in dairy animals is necessary.

Berberine is a natural plant alkaloid present in the roots, rhizomes, and stem bark of many traditional herbal plants. It has important bioactivities, such as the maintenance of lipid and glucose levels, modulation of immunity and insulin sensitivity, and broad spectrum of antimicrobial activities against a variety of microorganisms, including bacteria and chlamydia. To date, the predominant medical uses of berberine include the control of intestinal infections, diabetes, and cholesterol (Derosa et al., 2012; Tillhon et al., 2012). Increasing evi- 
dence suggests the potential antimicrobial properties of berberine against various bacterial pathogens, including MRSA and multi-drug resistant (MDR) S. epidermidis isolates (Wang et al., 2009a,b; Xu et al., 2009), suggesting that it may be a promising agent for preventing and treating mastitis in animal practice when used alone or in combination with commonly used antibiotics. The objectives of this study were to explore the antimicrobial activity of berberine alone or in combination with commonly used antibiotics in veterinary practice, such as penicillin, lincomycin, or amoxicillin, against $S$. epidermidis MDR field isolates from mastitis in dairy cows.

\section{MATERIAL AND METHODS}

\section{Bacterial strains}

Eighteen S. epidermidis field isolates were isolated from the milk of dairy cows that were diagnosed with clinical mastitis, provided by the Center of Animal Disease Control and Prevention of the Ningxia Autonomous Region, China (Yinchuan, China) (Table 1 and 2). The reference strain of S. epidermidis (FDA strain Seattle 1946, ATCC 12228) was obtained from the American Type Culture Collection (Manassas, VA, USA).

\section{Chemicals}

Chemicals used in this study were from Sigma (St. Louis, MO, USA), unless otherwise indicated. Muller-Hinton (MH) agar and MH broth were from Oxoid (Hampshire, UK). Lincomycin, amoxicillin, penicillin, and berberine were purchased from Pharmaceutical and Biological Products Inc. (Beijing, China). Lincomycin stock was freshly prepared as $1024 \mu \mathrm{g} /$ $\mathrm{mL}$ solution in phosphate buffer (PB), $\mathrm{pH} 4.5$; amoxicillin and penicillin were freshly prepared before use as stock solutions of $512 \mu \mathrm{g} / \mathrm{mL}$ in $\mathrm{PB}, \mathrm{pH} 6.5$; berberine stock was prepared as a solution with a concentration of $2048 \mu \mathrm{g} / \mathrm{mL}$ in $\mathrm{PB}, \mathrm{pH} 5.5$.

\section{In vitro determination of antibacterial susceptibility}

The methicillin resistance of the $S$. epidermidis isolate was determined as an assay for MRSA following the NCCLS guideline using $30 \mu \mathrm{g}$ cefoxitin and oxacillin. An isolate with an inhibition zone diameter of oxacillin inhibition $\leq 10 \mathrm{~mm}$, or cefoxitin $\leq 19 \mathrm{~mm}$ was considered to be a methicillin-resistant isolate (NCCLS, 2004). The antibacterial activities against the above $S$. epidermidis isolates were tested using an MH broth microdilution checkerboard method as previously described (Sun et al., 2009). Briefly, the bacterial culture was subcultured on $\mathrm{MH}$ agar and incubated at $37^{\circ} \mathrm{C}$ overnight. Single colonies were picked and inoculated into $\mathrm{MH}$ broth. The inoculum was adjusted to $1 \times 10^{6}$ colony-forming units (CFU)/ $\mathrm{mL}$ by comparison with a $0.5 \mathrm{X}$ McFarland turbidity standard. Next, $100 \mu \mathrm{L}$ bacterial suspension containing approximately $1 \times 10^{5} \mathrm{CFU}$ was used to test susceptibility against berberine, penicillin, lincomycin, and amoxicillin alone, and in the following combinations: berberine and penicillin, lincomycin, or amoxicillin. After cultivation at $37^{\circ} \mathrm{C}$ for $18 \mathrm{~h}$ under a microaerophilic atmosphere $\left(10 \% \mathrm{O}_{2} ; 5 \% \mathrm{CO}_{2}\right)$, the minimum inhibitory concentrations (MICs) were read as the minimum concentrations of drugs that completely inhibited the visible growth of microorganisms. The final drug concentration ranges were $1024-0.125 \mu \mathrm{g} / \mathrm{mL}$ for penicil- 
lin, lincomycin, and amoxicillin and $1024-0.5 \mu \mathrm{g} / \mathrm{mL}$ for berberine. The experiments were repeated a minimum of 3 times.

\section{Determination of interactions of drug combinations}

The interaction between berberine and penicillin, lincomycin, or amoxicillin was determined by evaluating the fractional inhibitory concentration index (FICI) as previously described (Oo et al., 2010). Briefly, MICs obtained from the checkerboard were further analyzed for FICIs based on the interaction theory of Loewe additivity (LA). The LA theory is based on the idea that a drug cannot interact with itself. FICI was determined from the MICs for each combination and was calculated using the following equation: FICI $=F I C A+$ FICB, where $F I C A=\mathrm{MIC}$ of drug A in combination with drug B/MIC of drug A alone, and FICB $=$ MIC of drug $\mathrm{B}$ in combination with drug $\mathrm{A} / \mathrm{MIC}$ of drug $\mathrm{B}$ alone. The FICI was interpreted as follows: FICI $\leq 0.5$, synergistic effect; $0.5<$ FITC $\leq 4.0$, no difference; FICI $>4.0$, antagonistic effect (Oo et al., 2010). All experiments were repeated 3 times.

\section{Time-kill assay}

A time-kill assay was conducted using the method described in the CLSI guidelines (CLSI, 2005; Credito et al., 2007). S. epidermidis isolate 4 was subjected to a further time-killing assay. Sub-inhibitory concentrations of drugs (alone and in combination) were added to tubes $(16 \mu \mathrm{g} / \mathrm{mL}$ penicillin and $128 \mu \mathrm{g} / \mathrm{mL}$ berberine; $64 \mu \mathrm{g} / \mathrm{mL}$ lincomycin and $32 \mu \mathrm{g} / \mathrm{mL}$ berberine; $64 \mu \mathrm{g} / \mathrm{mL}$ amoxicillin and $128 \mu \mathrm{g} / \mathrm{mL}$ berberine). A control tube without antibiotics was included in each series. In all cases, 1 drug in the combination did not affect the microorganism's growth curves when used alone. Tubes were inoculated with $\mathrm{MH}$ broth containing $10^{6} \mathrm{CFU} S$. epidermidis isolate 4 per $\mathrm{mL}$ and incubated at $37^{\circ} \mathrm{C}$ in a shaking incubator. Next, $100 \mu \mathrm{L}$ culture was removed from each tube at $0,4,8,12$, and $24 \mathrm{~h}$ after inoculation. Viability counts were conducted on Sabouraud dextrose agar plates after incubation at $35^{\circ} \mathrm{C}$ for $18 \mathrm{~h}$. A synergistic interaction was defined as a $2 \log _{10}$ decrease of $\mathrm{CFU} / \mathrm{mL}$ between the drug combination and its most active constituent. An antagonistic interaction was defined as a $2 \log _{10}$ increase in CFU/mL compared with a more active agent of the combination. A change of less than $2 \log _{10} \mathrm{CFU} / \mathrm{mL}$ was considered to indicate no difference. Each test was repeated at least 3 times.

\section{Statistical analysis}

Data are reported as means \pm standard deviation. The Student $t$-test was used to compare the means between the 2 groups. One-way analysis of variance was used to compare the means between more than 2 groups, and the comparison between 2 means of multiple groups was based on homogeneity of variance. The SPSS 13.0 software was used for the statistical analysis (SPSS, Inc., Chicago, IL, USA), and $\mathrm{P}<0.05$ indicated a significant difference.

\section{RESULTS}

\section{Methicillin-resistant S. epidermidis test}

The methicillin resistance of the $18 \mathrm{~S}$. epidermidis field isolates was determined fol- 
lowing the NCCLS guidelines for MRSA (NCCLS, 2004). Isolate number 4 of S. epidermidis (designated as $S$. epidermidis 4 in this study) was the only isolate resistant to methicillin (cefoxitin inhibition zone diameter $=12 \mathrm{~mm}$ ), which was chosen for further analysis using the time-kill assay as described below.

\section{Susceptibility of $S$. epidermidis isolates to antimicrobial agents}

The drug susceptibility of the 18 S. epidermidis isolates to berberine, penicillin, lincomycin, and amoxicillin was determined using a microdilution checkerboard assay (Table 1). These field strains isolated from the milk of dairy cows with mastitis were resistant to penicillin $(18 / 18,100 \%)$, lincomycin $(18 / 18,100 \%)$, amoxicillin (12/18, 66.7\%), and/or methicillin (1/18, $5.6 \%$ ). The MICs of berberine against these isolated ranged from $2-512 \mu \mathrm{g} / \mathrm{mL}$, while the MIC to the control S. epidermidis strain was $2 \mu \mathrm{g} / \mathrm{mL}$ (Table 1); the MICs of penicillin, lincomycin, and amoxicillin to $S$. epidermidis clinical isolates ranged from 0.8-213, 10-1024, and 0.4-256 $\mu \mathrm{g} /$ $\mathrm{mL}$, respectively; while the respective MICs of penicillin, lincomycin, and amoxicillin against the reference strain were $0.125,0.5$, and $0.5 \mu \mathrm{g} / \mathrm{mL}$ (Table 1). These results suggest that berberine has antibacterial activity against MDR S. epidermidis clinical isolates. In addition, the combinations of berberine with each of the tested antibiotics showed a decreased MIC against the microorganisms tested by 4-16-fold relative to the corresponding agents alone.

Table 1. Susceptibilities of Staphylococcus epidermidis field isolates to commonly used antibiotics.

\begin{tabular}{|c|c|c|c|c|c|c|}
\hline \multirow[t]{3}{*}{ S. epidermidis isolates } & \multicolumn{6}{|c|}{ Median MIC $(\mu \mathrm{g} / \mathrm{mL})$} \\
\hline & \multicolumn{3}{|c|}{ Alone } & \multicolumn{3}{|c|}{ In combination with berberine $(32 \mu \mathrm{g} / \mathrm{mL})$} \\
\hline & Penicillin & Lincomycin & Amoxicillin & Penicillin & Lincomycin & Amoxicillin \\
\hline S. epidermidis 1 & $2.33 \pm 1.53$ & $53.33 \pm 18.48$ & $0.83 \pm 0.29$ & $0.21 \pm 0.07^{* *}$ & $10.67 \pm 4.62 * *$ & $0.29 \pm 0.19^{* *}$ \\
\hline S. epidermidis 2 & $2 \pm 0$ & $106.67 \pm 36.95$ & $0.42 \pm 0.14$ & $0.25 \pm 0 * *$ & $16 \pm 0 * *$ & $0.21 \pm 0.07 * *$ \\
\hline S. epidermidis 3 & $5.33 \pm 2.31$ & $53.33 \pm 18.48$ & $2.67 \pm 1.15$ & $0.67 \pm 0.29 * *$ & $6.67 \pm 2.31 * *$ & $0.25 \pm 0 * *$ \\
\hline S. epidermidis 4 & $213.33 \pm 73.90$ & $1024 \pm 0$ & $256 \pm 0$ & $21.33 \pm 9.24 * *$ & $85.33 \pm 36.95^{* *}$ & $26.67 \pm 9.24 * *$ \\
\hline S. epidermidis 5 & $4 \pm 0$ & $42.67 \pm 18.48$ & $128 \pm 0$ & $1 \pm 0 * *$ & $4.67 \pm 3.06^{* *}$ & $42.67 \pm 18.48^{* *}$ \\
\hline S. epidermidis 6 & $106.67 \pm 36.95$ & $18.67 \pm 12.22$ & $128 \pm 0$ & $13.33 \pm 4.62 * *$ & $1 \pm 0^{* *}$ & $26.67 \pm 9.24 * *$ \\
\hline S. epidermidis 7 & $10.67 \pm 4.62$ & $53.33 \pm 18.48$ & $10.67 \pm 4.62$ & $1.33 \pm 0.58 * *$ & $4 \pm 0 * *$ & $1.17 \pm 0.76^{* *}$ \\
\hline S. epidermidis 8 & $6.67 \pm 2.31$ & $128 \pm 0$ & $1 \pm 0$ & $0.88 \pm 0.99 * *$ & $13.33 \pm 4.62 * *$ & $0.21 \pm 0.14^{* *}$ \\
\hline S. epidermidis 9 & $10.67 \pm 4.62$ & $42.67 \pm 18.48$ & $2.67 \pm 1.15$ & $2 \pm 0 * *$ & $5.33 \pm 2.31 * *$ & $1 \pm 0^{*}$ \\
\hline S. epidermidis 10 & $1.33 \pm 0.58$ & $10.67 \pm 4.62$ & $4.67 \pm 3.06$ & $0.25 \pm 0 * *$ & $3.33 \pm 1.15^{*}$ & $1.33 \pm 0.58$ \\
\hline S. epidermidis 11 & $128 \pm 0$ & $21.33 \pm 9.24$ & $256 \pm 0$ & $21.33 \pm 9.24 * *$ & $2 \pm 0 * *$ & $32 \pm 0 * *$ \\
\hline S. epidermidis 12 & $16 \pm 0$ & $64 \pm 0$ & $9.33 \pm 6.11$ & $2.67 \pm 1.15^{* *}$ & $9.33 \pm 6.11^{* *}$ & $0.5 \pm 0 * *$ \\
\hline S. epidermidis 13 & $0.83 \pm 0.29$ & $53.33 \pm 18.48$ & $8.67 \pm 7.02$ & $0.21 \pm 0.07^{* *}$ & $16 \pm 0^{* *}$ & $0.5 \pm 0^{* *}$ \\
\hline S. epidermidis 14 & $1.33 \pm 0.58$ & $16 \pm 0$ & $1.33 \pm 0.58$ & $0.25 \pm 0 * *$ & $4 \pm 0 * *$ & $0.17 \pm 0.07 * *$ \\
\hline S. epidermidis 15 & $128 \pm 0$ & $256 \pm 0$ & $4 \pm 0$ & $16 \pm 0 * *$ & $26.67 \pm 9.24 * *$ & $1 \pm 0 * *$ \\
\hline S. epidermidis 16 & $106.67 \pm 36.95$ & $512 \pm 0$ & $106.67 \pm 36.95$ & $10.67 \pm 4.62 * *$ & $64 \pm 0^{* *}$ & $13.33 \pm 4.62 * *$ \\
\hline S. epidermidis 17 & $128 \pm 0$ & $32 \pm 0$ & $128 \pm 0$ & $13.33 \pm 4.62 * *$ & $6.67 \pm 2.31 * *$ & $16 \pm 0 * *$ \\
\hline S. epidermidis 18 & $64 \pm 0$ & $37.33 \pm 24.44$ & $26.67 \pm 9.24$ & $8 \pm 0 * *$ & $2 \pm 0 * *$ & $6 \pm 3.46^{* *}$ \\
\hline ATCC 12228 & $0.125 \pm 0$ & $0.5 \pm 0$ & $0.5 \pm 0$ & $0.125 \pm 0$ & $0.125 \pm 0^{*}$ & $0.125 \pm 0^{*}$ \\
\hline
\end{tabular}

Susceptible or resistant strains were identified according to the interpretive breakpoints of CLSI criteria for penicillin $(<0.25$ and $\geq 0.25 \mu \mathrm{g} / \mathrm{mL}$, respectively); for lincomycin and amoxicillin $(<4$ and $\geq 4 \mu \mathrm{g} / \mathrm{mL}$, respectively) (CLSI, M100-S15, 2005). Results are reported as means \pm standard deviation for 3 independent experiments for each condition. $* \mathrm{P}<0.05,{ }^{*} * \mathrm{P}<0.01$ compared to the agent alone.

\section{Interactive effect of berberine and antibiotics against $S$. epidermidis isolates}

To investigate whether a combination of berberine and antibiotics would yield a syn- 
ergistic effect against $S$. epidermidis isolates, the FICI was calculated based on the above MIC data. As shown in Table 2, synergistic effects between berberine and penicillin were observed in most of the isolates tested. Such synergistic interactions were also found when berberine was used in combination with lincomycin or amoxicillin (data not shown). In contrast, such an interaction was not detected in the control strain of S. epidermidis. For the control S. epidermidis strain, combination treatment exhibited no interaction. Notably, no antagonistic effect was observed with any combinations for any of the strains tested.

Table 2. In vitro interactions between berberine and penicillin against Staphylococcus epidermidis field isolates

\begin{tabular}{lcccc}
\hline S. epidermidis isolates & \multicolumn{3}{c}{ Median (range) of FICIs } & \\
\cline { 2 - 5 } & FICI penicillin & FICI berberine & FICI combined & Interpretation \\
\hline S. epidermidis 1 & $0.104(0.063-0.125)$ & $0.25(0.25)$ & $0.354(0.188-0.625)$ & SYN \\
S. epidermidis 2 & $0.125(0.125)$ & $0.25(0.25)$ & $0.375(0.25-0.65)$ & SYN \\
S. epidermidis 3 & $0.125(0.125)$ & $0.208(0.125-0.25)$ & $0.333(0.25-0.375)$ & SYN \\
S. epidermidis 4 & $0.104(0.063-0.125)$ & $0.25(0.25)$ & $0.354(0.313-0.375)$ & SYN \\
S. epidermidis 5 & $0.25(0.25)$ & $0.25(0.25)$ & $0.5(0.375-0.75)$ & IND \\
S. epidermidis 6 & $0.125(0.125)$ & $0.104(0.063-0.125)$ & $0.229(0.188-0.25)$ & SYN \\
S. epidermidis 7 & $0.125(0.125)$ & $0.125(0.125)$ & $0.25(0.25)$ & SYN \\
S. epidermidis 8 & $0.13(0.016-0.25)$ & $0.104(0.063-0.125)$ & $0.234(0.188-0.375)$ & SYN \\
S. epidermidis 9 & $0.208(0.125-0.25)$ & $0.25(0.125-0.5)$ & $0.458(0.375-0.625)$ & SYN \\
S. epidermidis 10 & $0.208(0.125-0.25)$ & $0.25(0.25)$ & $0.458(0.375-0.5)$ & SYN \\
S. epidermidis 11 & $0.167(0.125-0.25)$ & $0.875(0.125-2)$ & $0.417(0.25-2.125)$ & IND \\
S. epidermidis 12 & $0.167(0.125-0.25)$ & $0.25(0.25)$ & $0.323(0.281-0.375)$ & SYN \\
S. epidermidis 13 & $0.25(0.25)$ & $0.073(0.31-0.125)$ & $0.875(0.75-1.125)$ & SYN \\
S. epidermidis 14 & $0.208(0.125-0.25)$ & $0.667(0.5-1.0)$ & $0.188(0.188)$ & IND \\
S. epidermidis 15 & $0.125(0.125)$ & $0.063(0.063)$ & $0.208(0.125-0.25)$ & SYN \\
S. epidermidis 16 & $0.104(0.063-0.125)$ & $0.104(0.063-0.125)$ & $0.354(0.313-0.375)$ & SYN \\
S. epidermidis 17 & $0.104(0.063-0.125)$ & $0.25(0.25)$ & $0.417(0.25-0.625)$ & SYN \\
S. epidermidis 18 & $0.125(0.125)$ & $0.292(0.125-0.5)$ & $1.25(1.25)$ & SYN \\
ATCC12228 & $1(1)$ & $0.25(0.25)$ & IND \\
\hline
\end{tabular}

SYN, synergism; IND, indifference. An FICI $<0.5$ was defined as a synergistic interaction, $0.5 \leq$ FICI $<4$ was defined as no interaction (no difference), and an FICI $\leq 4.0$ was defined as an antagonistic interaction. Results represent the mean and range for 3 independent experiments for each condition.

\section{Time-kill analysis}

Isolates of $S$. epidermidis 4 were resistant to penicillin, lincomycin, amoxicillin, and methicillin, and was thus chosen for further synergy studies using a time-killing assay with a combination of berberine with penicillin, lincomycin, or amoxicillin. Synergy time-kill graphs for $S$. epidermidis 4 are shown in Figure 1. The time-kill curves verified the synergistic effect for the combination of berberine and penicillin (Figure 1A), lincomycin (Figure 1B), or amoxicillin (Figure 1C). After $18 \mathrm{~h}$ of incubation, synergistic interactions were observed for berberine with the antibiotics, yielding a greater than $2 \log _{10} \mathrm{CFU} / \mathrm{mL}$ decrease in microorganisms, as compared with the most active agent, which was significantly greater than each drug alone $(\mathrm{P}<0.01)$. 


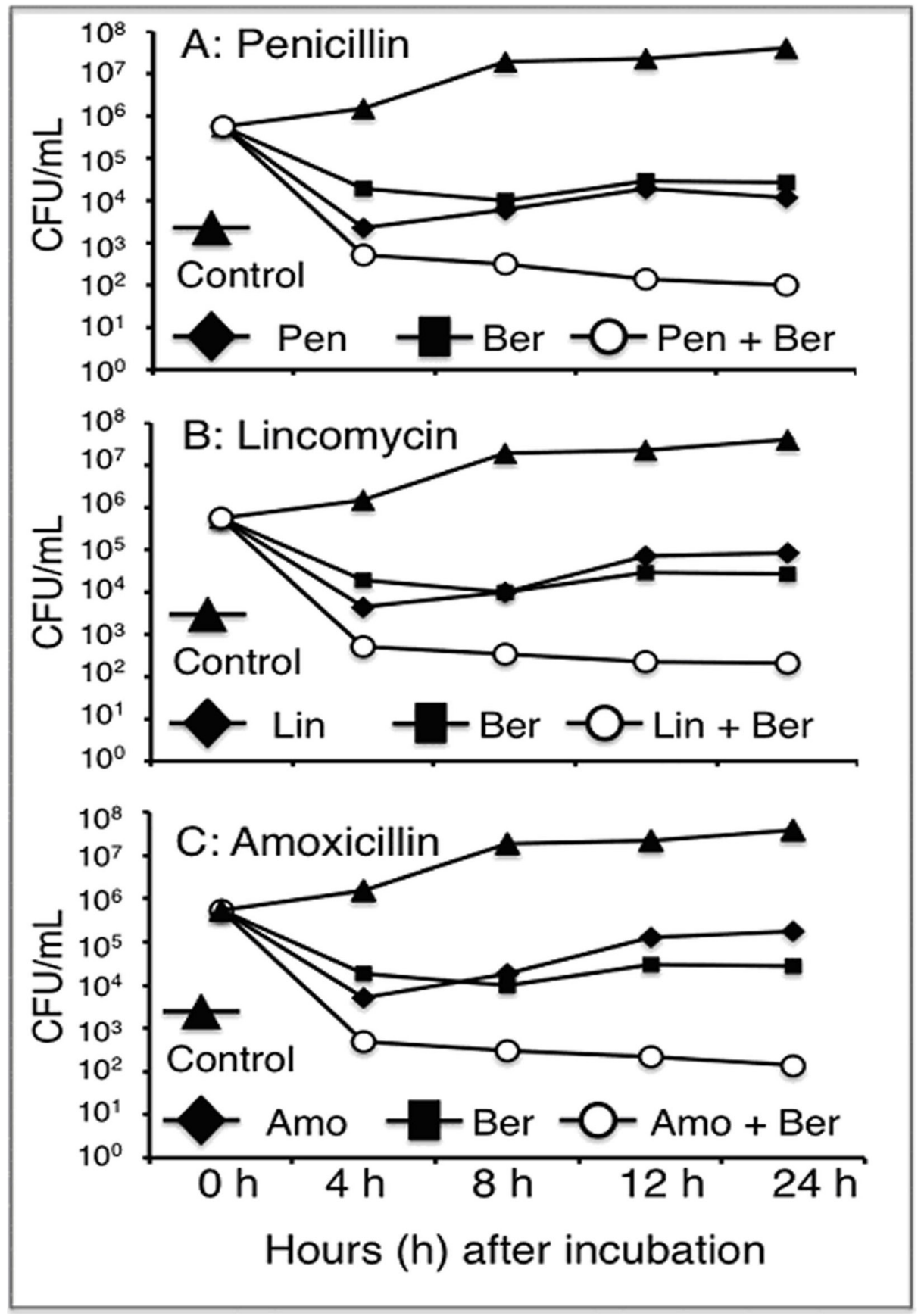

Figure 1. Time-kill curves for antimicrobial agents. Berberine and penicillin (A), lincomycin (B), or amoxicillin (C) were tested alone and in combination against Staphylococcus epidermidis 4, a field isolate resistant to penicillin, lincomycin, amoxicillin, and methicillin, using time-kill analysis. Results are reported as means \pm standard deviation for 3 independent experiments for each condition. 


\section{DISCUSSION}

In this study, the in vitro antibacterial activity of berberine against MDR S. epidermidis field isolates, either alone or in combination with penicillin, lincomycin, or amoxicillin, was explored. The results demonstrated the potential anti-staphylococcal activity of berberine at MICs of 2-512 $\mu \mathrm{g} / \mathrm{mL}$ against MDR S. epidermidis isolates. In combination with commonly used antibiotics, the susceptibility of most Staphylococcus strains tested compared to these agents alone was dramatically enhanced. This suggests a synergistic interaction between berberine and the antibiotics tested, which was also verified by the results of the time-kill assay. This study suggests that using a combination of existing antibiotics and natural herbal compounds will provide new means of optimal clinical effects, thus increasing efficacy and abating adverse drug reactions. Such a strategy may postpone the development of drug-resistance and enhance the clinical effects of commonly used antimicrobial agents.

CNS are Gram-positive cocci and include flora from the environment (such as Staphylococcus xylosus) and teat skin (such as S. epidermidis); they are capable of infecting dairy cows and heifers before calving and are important pathogens in cow mastitis with a higher prevalence in primiparous animals. In general, most CNS species are susceptible to commonly used antibiotics for mastitis treatment. However, different antimicrobial susceptibilities and diverse virulence factors were also found in CNS species isolated from mastitis in dairy cows (Taponen and Pyörälä, 2009); this may lead to ineffective treatment when using common antibiotics. In addition, an increasing number of CNS has been reported to be resistant to neomycin, penicillin, tetracycline, streptomycin, lincomycin, and ampicillin (Bochniarz and Wawron, 2011; Onni et al., 2011). Further, MDR isolates of S. epidermidis have been identified in the milk from both cows (Sawant et al., 2009; Waller et al., 2011) and women (Delgado et al., 2009), making these isolates a significant threat to public health. Consistent with these findings, most $S$. epidermidis isolates were determined to varying extents of MDR to penicillin, lincomycin, and/or amoxicillin in this study. All $18(100 \%)$ isolates tested were resistant to penicillin and licomycin, and/or 12 of $18(66.7 \%)$ were resistant to amoxicillin, although only 1 isolate (1/18), S. epidermidis 4, was methicillin-resistant (5.6\%).

An increasing number of studies have demonstrated that numerous herbs have antimicrobial properties, including anti-staphylococcal activity, and herbal medicines were less inclined to induce resistance in the clinical setting. Many lines of evidence have demonstrated the antimicrobial activity of berberine against a variety of bacterial pathogens including MRSA and S. epidermidis isolates (Wang et al., 2009a; Yu et al., 2010); berberine showed the potential to inhibit $S$. epidermidis biofilm formation in a previous study (Wang et al., 2009a). Importantly, a combination of berberine and other antimicrobial agents also had a synergistic effect against various pathogens, and such synergisms were observed when used in combination with fluconazole (Xu et al., 2009), miconazole (Wei et al., 2011), itraconazole (Lei et al., 2011), ampicillin, or oxicillin (Yu et al., 2010). The addition of berberine significantly decreased the MICs of these antimicrobial agents against the microorganisms tested compared with these agents alone. For example, a synergistic effect was observed between berberine and oxacillin against MRSA, and berberine showed a synergistic effect by increasing activity of oxacillin while decreasing MIC against MRSA. These results suggest that berberine may restore the effectiveness of beta-lactam antibiotics against MRSA (Yu et al., 2010). Additionally, the anti-staphylococcal activity of berberine was also observed in this study. More importantly, a combination of berberine and antibiotics, including penicillin, lincomycin, and 
amoxicillin yielded a synergistic effect against MDR S. epidermidis field isolates. These antimicrobial properties of berberine may be useful for controlling and treating mastitis caused by MDR Staphylococcus infection in dairy cows.

\section{CONCLUSIONS}

Our results demonstrate that berberine has antimicrobial properties against MDR $S$. epidermidis strains. Particularly, a favorable synergistic interaction was observed when berberine was used in combination with commonly used antibiotics, including penicillin, lincomycin, and amoxicillin against MDR Staphylococcus field isolates. These findings indicate that berberine can be used for the prevention and treatment of Staphylococcus causing mastitis in dairy cows, either alone or in combination with other antimicrobial agents.

\section{ACKNOWLEDGMENTS}

Research supported by grants from the National Natural Science Foundation of China (\#31260624 and \#31060348), and a grant from the National Science Technology Support Program of China (\#2012BAD12B07-4).

\section{REFERENCES}

Bochniarz M and Wawron W (2011). Antibiotic susceptibility of methicillin-resistant and methicillin-susceptible coagulase-negative staphylococci isolated from bovine mastitis. Pol. J. Vet. Sci. 14: 405-410.

CLSI (2005). Performance standards for antimicrobial susceptibility testing. Clinical and Laboratory Standards Institute, 15th Informational Supplement, Wayne, PA, Untied States, M100-S115.

Credito K, Lin B and Appelbaum PC (2007). Activity of daptomycin alone and in combination with rifampin and gentamicin against Staphylococcus aureus assessed by time-kill methodology. Antimicrob. Agents Chemother. 51: 1504-1507.

Delgado S, Arroyo R, Jiménez E, Marín ML, et al. (2009). Staphylococcus epidermidis strains isolated from breast milk of women suffering infectious mastitis: potential virulence traits and resistance to antibiotics. BMC Microbiol. 9: 82.

Derosa G, Maffioli P and Cicero AF (2012). Berberine on metabolic and cardiovascular risk factors: an analysis from preclinical evidences to clinical trials. Expert Opin. Biol. Ther. 12: 1113-1124.

Fernández-Rufete A, García-Vázquez E, Hernández-Torres A, Canteras M, et al. (2012). Coagulase-negative Staphylococcus bacteraemia: prognosis factors and influence of antibiotic treatment. Rev. Esp. Quimioter. 25: 199-205.

Gomes F, Teixeira P, Ceri H and Oliveira R (2012). Evaluation of antimicrobial activity of certain combinations of antibiotics against in vitro Staphylococcus epidermidis biofilms. Indian J. Med. Res. 135: 542-547.

Holmes MA and Zadoks RN (2011). Methicillin resistant S. aureus in human and bovine mastitis. J. Mammary Gland Biol. Neoplasia 16: 373-382.

Koop G, De Vliegher S, De Visscher A, Supré K, et al. (2012). Differences between coagulase-negative Staphylococcus species in persistence and in effect on somatic cell count and milk yield in dairy goats. J. Dairy Sci. 95: 5075-5084.

Lei G, Dan H, Jinhua L, Wei Y, et al. (2011). Berberine and itraconazole are not synergistic in vitro against Aspergillus fumigatus isolated from clinical patients. Molecules 16: 9218-9233.

Mekni MA, Bouchami O, Achour W and Ben Hassen A (2012). Strong biofilm production but not adhesion virulence factors can discriminate between invasive and commensal Staphylococcus epidermidis strains. APMIS 120: 605-611.

NCCLS (2004). Performance Standards for Antimicrobial Disk Susceptibility Tests: Approved Standard M2-A7. National Committee for Clinical Laboratory Standards, Wayne.

Nickerson SC (2009). Control of heifer mastitis: antimicrobial treatment-an overview. Vet. Microbiol. 134: 128-135.

Oliveira M, Bexiga R, Nunes SF, Carneiro C, et al. (2006). Biofilm-forming ability profiling of Staphylococcus aureus and Staphylococcus epidermidis mastitis isolates. Vet. Microbiol. 118: 133-140.

Onni T, Sanna G, Larsen J and Tola S (2011). Antimicrobial susceptibilities and population structure of Staphylococcus epidermidis associated with ovine mastitis. Vet. Microbiol. 148: 45-50.

Oo TZ, Cole N, Garthwaite L, Willcox MD, et al. (2010). Evaluation of synergistic activity of bovine lactoferricin with antibiotics in corneal infection. J. Antimicrob. Chemother. 65: 1243-1251. 
Piessens V, De Vliegher S, Verbist B, Braem G, et al. (2012). Intra-species diversity and epidemiology varies among coagulase-negative Staphylococcus species causing bovine intramammary infections. Vet. Microbiol. 155: 62-71.

Pyörälä S and Taponen S (2009). Coagulase-negative staphylococci-emerging mastitis pathogens. Vet. Microbiol. 134: 3-8.

Saini V, McClure JT, Scholl DT, DeVries TJ, et al. (2012). Herd-level association between antimicrobial use and antimicrobial resistance in bovine mastitis Staphylococcus aureus isolates on Canadian dairy farms. J. Dairy Sci. 95: 1921-1929.

Sawant AA, Gillespie BE and Oliver SP (2009). Antimicrobial susceptibility of coagulase-negative Staphylococcus species isolated from bovine milk. Vet. Microbiol. 134: 73-81.

Sun L, Sun S, Cheng A, Wu X, et al. (2009). In vitro activities of retigeric acid B alone and in combination with azole antifungal agents against Candida albicans. Antimicrob. Agents Chemother. 53: 1586-1591.

Taponen S and Pyörälä S (2009). Coagulase-negative staphylococci as cause of bovine mastitis- not so different from Staphylococcus aureus? Vet. Microbiol. 134: 29-36.

Tillhon M, Guamán Ortiz LM, Lombardi P and Scovassi AI (2012). Berberine: new perspectives for old remedies. Biochem. Pharmacol. 84: 1260-1267.

Waller KP, Aspán A, Nyman A, Persson Y, et al. (2011). CNS species and antimicrobial resistance in clinical and subclinical bovine mastitis. Vet. Microbiol. 152: 112-116.

Wang X, Qiu S, Yao X, Tang T, et al. (2009a). Berberine inhibits Staphylococcus epidermidis adhesion and biofilm formation on the surface of titanium alloy. J. Orthop. Res. 27: 1487-1492.

Wang X, Yao X, Zhu Z, Tang T, et al. (2009b). Effect of berberine on Staphylococcus epidermidis biofilm formation. Int. J. Antimicrob. Agents 34: 60-66.

Wei GX, Xu X and Wu CD (2011). In vitro synergism between berberine and miconazole against planktonic and biofilm Candida cultures. Arch. Oral Biol. 56: 565-572.

Xu Y, Wang Y, Yan L, Liang RM, et al. (2009). Proteomic analysis reveals a synergistic mechanism of fluconazole and berberine against fluconazole-resistant Candida albicans: endogenous ROS augmentation. J. Proteome Res. 8: 5296-5304.

Yu YY, Wang H, Zhang SW and Wang BE (2010). Inhibition of methicillin-resistant Staphylococcus aureus by the compound Qingre granules. Chin. Med. J. (Engl.) 123: 1017-1020.

Zadoks RN, Middleton JR, McDougall S, Katholm J, et al. (2011). Molecular epidemiology of mastitis pathogens of dairy cattle and comparative relevance to humans. J. Mammary Gland Biol. Neoplasia 16: 357-372. 